\title{
P72 Vascular Aging Prediction in High Cardiovascular Risk Cohort
}

\author{
Agne Laucyte-Cibulskiene*, Ligita Ryliskyte, Milda Kovaite, Laurynas Rimsevicius, Laura Balkeviciene,
} Jolita Badariene, Aleksandras Laucevicius

Institute of Clinical Medicine, Faculty of Medicine, Vilnius University, Vilnius, Lithuania

\section{ABSTRACT}

Aim: To assess a time-dependent change of arterial stiffness gradient (PWVratio calculated by dividing carotid-femoral to carotid-radialPWV), a marker of vascular aging, in high cardiovascular risk cohort.

Methods: This cohort study $(n=689)$ was conducted during the period 2010-2019 at Vilnius University Hospital Santaros Klinikos. Participants were referred by the primary health care institutions according to Lithuanian High Cardiovascular Risk (LitHiR) primary prevention program which recruited men at the age of 40-55 years and women at the age of 50-65 years without overt cardiovascular disease; they underwent a detailed cardiovascular risk assessment and arterial stiffness evaluation and were repeatedly examined after the mean follow-up of $965.63 \pm 511.98$ days.

Results: The cohort was comprised of $40.2 \%$ male participants. Cardiovascular risk factors were distributed as follows: $19.5 \%$ subjects had diabetes mellitus, 52.69\% left ventricular hypertrophy, $95.21 \%$ hypertension, $99.13 \%$ dyslipidemia. During the follow-up period $52.1 \%$ of patients presented PWV ratio increase. In spite of the difference in age between men and women, PWV ratio progression adjusted for follow-up time and age was more pronounced in males (OR 1.72, $p=0.032$ ). In the subanalysis, we found that in men, as compared to women, there was a more pronounced decrease in carotid-radialPWV $(p<0.001)$. The carotid-femoralPWV increased both in men and in women. Glucose level, abdominal obesity, mean arterial blood pressure, menopause was related to the change of the PWV ratio.

Conclusion: In this high cardiovascular risk cohort, the change in arterial stiffness gradient is related to abdominal obesity, mean arterial blood pressure, and menopause.

(c) 2019 Association for Research into Arterial Structure and Physiology. Publishing services by Atlantis Press International B.V. This is an open access article distributed under the CC BY-NC 4.0 license (http://creativecommons.org/licenses/by-nc/4.0/). 